\title{
Linear Quadratic and Tumour Control Probability Modelling in External Beam Radiotherapy
}

\author{
SFC O'Rourke a,b,*
}

${ }^{a}$ School of Mathematics and Physics, Queen's University Belfast, Belfast, BT7 1NN, U.K.

${ }^{\mathrm{b}}$ Centre for Cancer Research and Cell Biology, School of Biomedical Sciences, Queen's University Belfast, Belfast BT9 7AB, U.K.

\author{
H McAneney \\ School of Mathematics and Physics, Queen's University Belfast, Belfast, BT7 1NN, U.K.
}

T Hillen

Department of Mathematical and Statistical Sciences, University of Alberta, Edmonton, Alberta, T6G 2G1, Canada

\begin{abstract}
The standard Linear-Quadratic (LQ) survival model for external beam radiotherapy is reviewed with particular emphasis on studying how different schedules of radiation treatment planning may be affected by different tumour repopulation kinetics. The LQ model is further examined in the context of tumour control probability (TCP) models. The application of the Zaider and Minerbo non-Poissonian TCP model incorporating the effect of cellular repopulation is reviewed. In particular the recent development of a cell cycle model within the original Zaider and Minerbo TCP formalism is highlighted. Application of this TCP cell-cycle model in clinical treatment plans is explored and analyzed.
\end{abstract}

Key words: fractionation, repopulation, BED

PACS:

\section{Introduction and Historical Background}

The purpose of this article is to review recent contributions in radiobiological modelling applied to external beam radiotherapy which concentrate on the role of cellular repopulation between treatments and cell-cycle effects which influence the outcome of treatment. Owing to the enormous body of theoretical and clinical publications devoted to radiobiological modelling, no such review could be com-

\footnotetext{
* Corresponding author

Email addresses: s.orourke@qub.ac.uk (SFC O'Rourke

), h.mcaneney@qub.ac.uk (H McAneney), thillen@math.ualberta.ca (T Hillen).
}

prehensive. Thus we will concentrate only on two of the most commonly used formalisms in radiotherapy. These are the Linear Quadratic (LQ) and Tumour Control Probability (TCP) models.

The plan of the article is as follows. Section 1.1 reviews the historical development of clinical applications of radiobiological modelling using the LQ and TCP formulae. Section 1.2 and 1.3 focuses on the theoretical development of the LQ and TCP models in cancer radiotherapy. Section 2 presents the radiobiological theory of the LQ and TCP models. Section 3 addresses the issue of repopulation within the LQ model and discusses recent developments within this particular formalism of the LQ model. Section 4 examines the important role of the cell-cycle within 
the TCP model and analyses how the recent models of Dawson and Hillen [1] enable the TCP to be calculated for general time-dependent treatment protocols. In Section 5 we discuss future directions yet to be explored within the context of LQ and TCP radiobiological modelling and present some concluding remarks.

\subsection{Clinical Applications of Radiobiological Modelling in External Beam Radiotherapy}

Major advances during the last fifty-five years have been made by radiobiologists in understanding the mechanisms of how radiation causes DNA damage. A notably robust mathematical model that has been adopted widely in radiation oncology is the LQ formalism [2-10]. This model predicts dose-time relationships and has been a commonly used model for studying cell survival analysis. The LQ model takes account of the two basic mechanisms of cell death or sterilization: repairable lesion exchange and nonrepairable lesion [11]. In addition, the LQ model incorporates one of the fundamental aims of radiation treatment, that of separating the responses of the tumour, early responding healthy tissue and late responding healthy tissue.

The LQ model has the practical advantage that it results in a simple analytical form for the survival fraction and can also be employed in the prediction of disease free survival probability TCP models. As such, it is applicable clinically to a wide range of external beam radiotherapy treatment schedules. Widely implemented clinical treatment schedules in electron beam radiation oncology include (see Table 1 for details): (i) Standard fractionation, [12-14] (ii) Hyper-fractionation (smaller dose per fraction, same total dose and overall treatment time) e.g. for oropharyngeal cancer, $[15,14,16]$, (iii) Accelerated fractionation (shorter overall treatment time with the same total dose) e.g. for head and neck cancer $[17,18]$ (iv) CHART (Continuous Hyperfractionated Accelerated Radiotherapy) for head and neck cancer [19,20], glioblastomas [21] and non-small lung carcinoma $[19,22]$. Other alternative schedules include: ARCON (Accelerated Hyperfractionated Radiation therapy with Carbogen and Nicotinamide) employed in laryngeal cancer $[14,13,23]$; SMART boost (Simultaneous Modulated Accelerated Radiation Therapy) used with success in the treatment of head and neck cancer, $[14,13,23]$; hypo-fractionation (a smaller number of larger-dose fractions) applied
Dose/frac. No. of Days/week Times/day Total dose

(Gy) fractions (\# of weeks) (interval)

\begin{tabular}{cccccc} 
i & 2 & $30-35$ & $5(6-7)$ & 1 & $60-70$ \\
ii & 1.15 & 70 & $5(7)$ & $2(4-6 \mathrm{hrs})$ & 80.5 \\
iii & 1.6 & 45 & $5\left(5^{*}\right)$ & 3 & 72 \\
iv & $1.4-1.5$ & 36 & $7(1.7)$ & $3(6 \mathrm{hrs})$ & $50-54$ \\
\hline
\end{tabular}

Table 1

* 2 week period of rest in middle

Typical implementation of various radiation treatment protocols. Roman numerals correspond to those protocols listed in text.

in the treatment of prostate cancer [24-26]; split course (intentional gaps in radiation therapy) $[14,13]$ and six days per week treatment protocols [27].

Mathematical and statistical modelling have played a crucial role in developing many of the above treatment schedules. They can give vital insight into whether a particular schedule maybe suitable or not to be used in a clinical setting. In the next section we review the different variants of the LQ model that have been developed with the aim of improving treatment outcome for cancer patients.

\subsection{Theoretical developments of the $L Q$ model in electron beam radiotherapy}

The most commonly used model for studying the survival response to radiotherapy is the LQ model $[28,29]$. This model considers the effect of both irreparable damage and repairable damage susceptible to misrepair which ultimately leads to mitotic cell death. The LQ model comes in various degrees of complexity depending on the number of the well established "5R's" of radiobiology that are incorporated into the model (that is, the 4 "R's" by Withers - Repair, Repopulation, Re-distribution and Re-oxygenation [30] and more recently intrinistic radioresistance [31]). Studies which extended the LQ model to account for exponential repopulation include Wheldon et al.[32], Usher [33], Travis and Tucker [34] and Fowler [29]. The effects of hypoxia have been addressed by Woulters and Brown [35] using a one-compartment model based on the assumption that oxygen is purely dose dependent. Brenner et al.[36] have considered a one-compartment model of the LQ model to take into account the effects of re-oxygenation and redistribution assuming a Gaussian distribution for 
the radiosensitivity parameters along with exponential repopulation. Two-compartment models (hypoxic and oxic), where re-oxygenation is represented by the flux of cells between the two compartments have been developed by Buffa et al.[37] and then extended by Horas et al. [38].

Optimisation of radiotherapy treatment within LQ modelling incorporating exponential repopulation has been studied by Wheldon et al.[32] and Wein et al.[39]. More recently, McAneney and O'Rourke integrated logistic and Gompertzian growth laws into the LQ model [40]. The LQ model has also been incorporated into 4D simulation models for tumour response to radiotherapy in vivo by Antipas et al.[41], Dionysiou et al.[42], Dionysiou and Stamatakos [21]. Another variant of the LQ model captures the process of the mitotic cycle [4346]. Other advances in fractionated radiotherapy include the effect of the delay on tumour repopulation during treatment [47]. However, of the 5 R's of radiotherapy that exist, it has emerged from clinical studies that repopulation is one of the most significant factors that can provide insight into the lack of efficacy of radiation treatment. Indeed, Kirkpatrick and Marks [48] stated that simple radiobiologic models that fail to incorporate the heterogeneity of radiosensitivity and/or tumor cell repopulation will not adequately describe clinical outcomes. In addition, the recent of Kim and Tannock[49] on repopulation of cancer cells during chemotherapy or radiation treatment also provides evidence to indicate that repopulation often has a dominant effect on treatment outcome. The kinetics of repopulation offer insight into the underlying mechanisms of tumour cell death and re-growth, and as such, these models may be clinically useful in predicting response to therapy [50].

These LQ models may also be used to design optimum treatment protocols in which the aim is to maximize tumour control for the minimum normaltissue complications. Optimum fractionation schedules depend critically on the proliferative nature of the tumour cells. Three clinical examples that illustrate this are, (i) head and neck cancer $[19,20]$, (ii) non-small cell lung cancer $[19,22,51]$ and (iii) prostate cancer [52].

In head and neck cancer and non-small cell lung cancer, the tumours proliferate so fast that shorter schedules such as CHART are required. Clearly, modelling a schedule for treatment based on the LQ model will be more accurate if repopulation effects are included based on the biological proliferation rate of the tumour. In prostate cancer, the tumours proliferate slowly which allows so much repair time between fractions that larger doses are required. Again, it is clear that including the repopulation kinetics here would enable clinicians to exploit optimization schedules to enhance treatment outcome for prostate cancer. It has been shown by Dionysiou et al.[42] that in a hyper-fractionation scheme for glioblatomas there is a marked decrease in repopulation compared to the standard fractionation normally used. This agrees with clinical studies which indicate that hyper-fractionation generally improves tumour control rates for aggressively proliferating tumours [29]. The debate about the importance of repopulation effects has led to other models with more specific growth laws being proposed to describe tumour proliferation and regrowth. These include the work of O'Donoghue[53], Wheldon et al. [54], Lindsay et al.[55], Mao et al. [56] and McAneney and O'Rourke[40]. These models are reviewed in section 3 were in particular we focus on examining the role that various non-linear growth laws have on the outcome of cancer radiotherapy treatment schedules.

\subsection{Theoretical development of TCP models within the $L Q$ formalism}

The LQ model has also been integrated with a time independent tumour control probability by Munro and Gilbert[57]. In their model they postulated that the distribution of clonogens after radiation treatment is represented by a Poisson distribution and obtained a simple statistical formula for disease free probability incorporating the survival fraction formula from the basic LQ model of cell damage and cell recovery. This model has been widely analyzed for application in clinical radiation treatment protocols [58-60]. Maciejewski et al.[18] have used this model to improve TCP outcome using accelerated schedules in head and neck cancer to minimize tumour repopulation during therapy. Horiot et al. [15] have used the TCP model aimed at improving outcome by increasing the overall dose delivered in hyperfractionation protocols in oropharyngeal cancer. But the limitations of this early model are widely acknowledged [61-63]. Indeed the binomial/Poisson formula always underestimates the TCP and this is one of its main flaws. Another flaw of Poisson model is that it neglects tumour clonogenic repopulation during therapy. Tucker 
and Taylor[64] obtained improvements upon the conventional Poisson TCP model by adopting a numerically based geometric stochastic approach to account for tumour cell repopulation. Kendal [63] has obtained an analogous closed analytic form of the numerical models proposed by Tucker and Taylor[64]. Later in 1999, Tucker improved the 1996 model to account for cell cycle effects, rate of cell differentiation and the cell rate loss.

Clonogen repopulation in Poissonian TCP calculations within the LQ model has also been accounted for by introducing a time-dependent term into the formalism [65,66,29,67-69]. Other Poissonian TCP model which extend the early TCP models to include radiobiological cellular responses (other than repopulation) have been considered in the literature. For example, a closed form expression for radiation control probability of hetergeneous tumours has been obtained by Fenwick[70]. TCP models have been developed by Nahum and Tait[71], Webb and Nahum[72], Brenner[60] and Webb[73] which incorporate the effect of distributions in the dose to the tumour and clonogenic cell density. Mohan et al. [74] have considered a TCP model for prediction of the cost function to be optimized in 3D treatment planning. Buffa et al.[37] have investigated the TCP model within a two compartment model for oxic and hypoxic tumour cells using a LQ formulation and an oxygen diffusion model.

The models discussed so far are Poissonian. This issue has been rectified by Zaider and Minerbo[75] who have developed a non-Poissonian dose-time dependent exact tumour control probability formula based on birth and death stochastic processes to include cellular repopulation. This important contribution corrects one of the flaws of the original time-independent TCP model based on the binomial/Poisson formula which results in underestimating the TCP. Based on the Zaider and Minerbo TCP formula which is valid for any temporal protocol of dose delivery Stravreva et al. [76] have derived a TCP formula specifically for external fractionated radiotherapy and shown this was applicable to the case of variable probability of cell kill per dose fraction. Dawson and HIllen[1] have extended the Zaider and Minerbo [75] TCP formulation to include the effects of the cell cycle. The TCP models of Zaider and Minerbo are reviewed in section 4.

Since the aim of radiotherapy is to maximize damage to the tumour but at the same time minimize damage to normal healthy tissue then it should be noted that TCP models are maximized subject to some upper limit on the allowed normal tissue complication probability (NTCP). Traditionally both TCP models and their corresponding NTCP model are used by clinicians to establish guidelines for radiotherapists to predict dose and best clinical practice for future patients. We do not intend to discuss NTCP models in depth in this review due to the scope of the article but refer the reader to well established and clinically accepted NTCP models in the literature $[77,78]$.

\section{Basics and radiobiological background of LQ modelling}

The LQ model was originally developed from biophysical considerations rather than empirical clinical observations and as such it is closely associated with parameters more likely to influence biological response. The mechanistic basis for the LQ model has been extensively reviewed in the literature by Sachs et al.[79], Brenner et al.[80] and Guerrero et $a l$.[81]. Derivation of the LQ model is not unique and has been obtained by many authors from different viewpoints [82-89]. The expression for the LQ model may be simply stated as

$\ln \sigma=-\alpha D-\beta G D^{2}$.

This expresses the surviving fraction of clonogenic cells $\sigma$ in terms of two parameters, $\alpha$ and $\beta$. The parameter $\alpha$ represents lethal lesions made by one track action and $\beta$ accounts for lethal lesions made by two-track action. $D$ is the radiation doses and $G$ is the Lea-Catcheside dose-protraction factor and is given by $[82,8,83]$

$G=2 \int_{0}^{T} \frac{R(t)}{D} d t \int_{0}^{t} \frac{R\left(t^{\prime}\right)}{D} e^{\lambda\left(t^{\prime}-t\right)} d t^{\prime}$,

where $D=D(T)$ is the total dose in the interval, $R(t)$ the time varying dose rate and $\lambda$ the repair time constant. This dose rate function, $G$, encompasses the temporal behaviour of radiation delivery in its entirety. Hence, Eq. 2 can be used to estimate the protraction effects in the following cases: (i) single fractionation delivered at a constant rate, (ii) split dose and multi-fraction irradiation protocols and (iii) continuous low dose rates encountered in brachytherapy. The protraction factor $G$ biophysically represents that a potentially lethal lesion (i.e. a double strand break) is created at time $t^{\prime}$ and if not repaired, may interact in a pairwise manner with a 
second lethal lesion produced at time $t[5]$. In the case of a constant dose rate, as one has in the situation for external beam radiotherapy, the dose rate $R(t)$ is defined by the following function,

$R(t)=\left\{\begin{array}{l}\frac{D}{T} t \in[0, T] \quad T>0 \\ 0 \text { else }\end{array}\right.$

from which we can then calculate $G$ using Eq. 2. Thus we obtain

$$
\begin{aligned}
G & =\frac{2}{T^{2}} \int_{0}^{T} \int_{0}^{t} e^{\lambda\left(t^{\prime}-t\right)} d t^{\prime} d t \\
& =\frac{2}{(\lambda T)^{2}}\left(\lambda T+e^{-\lambda T}-1\right) .
\end{aligned}
$$

$T$ is the irradiation duration time. If the irradiation time is short enough, the term $\lambda T$ in the above equation tends to zero. The exponential term can be expanded using a Taylor's series and by neglecting terms of order $(\lambda T)^{3}$ in the Taylor series approximation it is found that $G \rightarrow 1$. However, if irradiation treatment is prolonged, such as in the case of continuous low radiation schedules that are typically used in brachytherapy, then $G<1$, since the kernel $\exp \left[\left(t^{\prime}-t\right)\right] \leq 1$ for $t^{\prime} \leq t[8]$.

For the remainder of this article we are only concerned with normal external beam radiotherapy where the duration of delivering a fraction is measured in seconds and the repair time constant is typically an hour. In this case $G(t)$ is effectively equal to unity as shown above and the dose referred to as an 'acute' dose. In the case of fractionated schedules where the dose is given daily and there is no interaction between the schedules, then it follows that after $n$ fractions each of dose $d$, the final survival fraction arising from each of the individual fractions is

$\sigma=e^{-\alpha n d-\beta n d^{2}}=e^{-(\alpha+\beta d) D}$,

where the total dose $D=n d$. This formalism presumes complete cellular repair between treatments and can be extended to incorporate cellular repopulation using the logistic or Gompertz laws. This will be discussed in section 3 and compared with existing repopulation models using a time-dependent factor.

\subsection{Fractionation sensitivities: $\alpha / \beta$ ratios}

In the LQ model the ratio $(\alpha / \beta)$ is an inverse measure of a tissue's sensitivity to fractionation, that is,

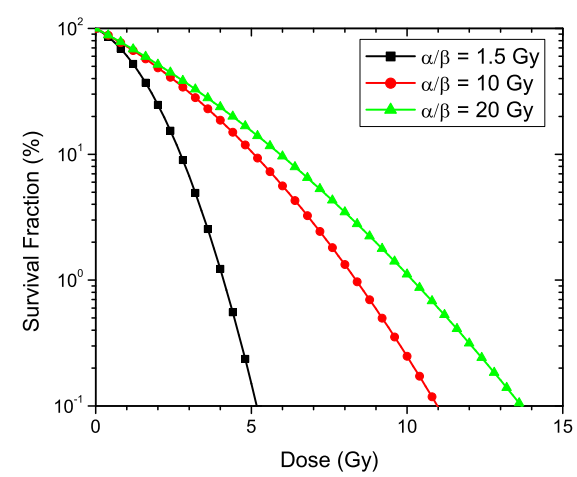

Fig. 1. Cell survival curves illustrating the surviving fraction of cells after a single dose of radiation. The cases shown are for prostate cancer $(\alpha / \beta=1.5 \mathrm{~Gy})$, non-small cell cancer $(\alpha / \beta=10 \mathrm{~Gy})$ and advanced head and neck cancer $(\alpha / \beta=20 \mathrm{~Gy})$.

the size of dose given on each treatment. For example, a typical value for $\alpha / \beta$ range between $3-10 \mathrm{~Gy}$ $[6,4,90]$. In fact, in the case of prostate cancer which is a very slowly proliferating, late responding tissue $\alpha / \beta$ can be as low as 1 Gy $[91,92]$. At the other end of the spectrum $\alpha / \beta$ may be as high as $20 \mathrm{~Gy}$ in the case of advanced head and neck cancer which is an early responding tissue with an extremely aggressive rate of cell proliferation $[13,90]$. Recent advances in treatment protocols have resulted from taking account of the particular radiobiological cell survival parameters $(\alpha / \beta)$ involved. The cell survival curves shown in Fig. 1 are plotted for values of $\alpha / \beta=1.5$, 10 and 20 for prostate cancer, non-small cell lung cancer and advanced head and neck cancer respectively. This range in values corresponds respectively from late responding tissue, which has a high repair capacity, to acute responding tissue which has a low repair capacity. Acute responding tissues have fast cellular turn over and therefore show signs of radiation induced damage to normal tissue days to weeks after exposure. This can be explained due to the short lifespan of their mature cells. By comparison late responding tissues show effects months to years later because they have a low level of cellular turnover and the interval between cell divisions is long giving the cells an opportunity to repair radiobiological damage [14]. Fraction size is a dominant feature in determining late effects with overall treatment time having little influence. In contrast, the response by acute responding tissue is influenced by (i) fractionation, but to a lesser degree, and (ii) the overall treatment time [14]. 


\subsection{Biological Effective Dose (BED)}

One of the main clinical applications of the LQ model is to calculate the total dose on a treatment regimen which would have the same effect on a given tissue as some other regimen. This concept is known as the biologically effective dose (BED) and was first introduced by Barendsen[93]. It was originally known as the extrapolated response dose (ERD) and later re-named to the present day terminology (BED) by Fowler[29]. In this section we only consider an application of the BED for well-spaced high dose fractions in Eq. 6 where the protraction factor $G$ is unity. The BED formula employed for clinical applications in external beam fractionated radiotherapy is given by

$B E D=-\frac{\ln (\sigma)}{\alpha}=D\left(1+\frac{d}{\alpha / \beta}\right)$

where $n$ is the number of fractions, $d$ is the dose per fraction and $D$ is the total dose delivered over the course of treatment. The term in brackets in the equation above is the relative effectiveness so that BED is total dose $\times$ relative effectiveness. The BED model represents the dose required for a given effect when delivered by infinitely small doses per fraction. To achieve isoeffectiveness between two fractionation schedules of total doses $D_{1}=n d_{1}$ and $D_{2}=$ $n d_{2}$ where $d_{1}$ and $d_{2}$ represent the doses per fraction respectively we obtain

$D_{1}\left(1+\frac{d_{1}}{\alpha / \beta}\right)=D_{2}\left(1+\frac{d_{2}}{\alpha / \beta}\right)$

and $\alpha / \beta$ ratios can be estimated if the parameters $n, d_{1}$ and $d_{2}$ are known. For any normal or tumour tissue, an increased BED indicates an increased biological effect. That is, a reduced surviving fraction, $\sigma$, for both normal and tumour cells. The goal of radiotherapy is to minimize damage to normal tissue and maximize damage to tumour tissue. In mathematical terms this means that for healthy tissue surrounding the tumour the aim is to maximize $\sigma$ in the case of normal tissue while simultaneously minimizing the value $\sigma$ for the tumour tissue. As larger values of $\beta$ imply an increased likelihood of potentially repairable ionizing events, it follows that tissues with smaller $\alpha / \beta$ ratios exhibit a greater dosesparing effect than do those with larger values of $\alpha / \beta$. That is, tissues with smaller $\alpha / \beta$ ratios have a larger surviving fraction $\sigma$ after treatment than tissues with a larger $\alpha / \beta$ ratio. Another factor to bear

\begin{tabular}{cccccc}
\hline & Total & Fraction & \multicolumn{3}{c}{ BED } \\
Dose $D$ & Dose $d$ & $n$ & $\mathrm{~Gy}_{3}$ & $\mathrm{~Gy}_{20}$ \\
\hline$*$ & 66.0 & 2.0 & 33 & 110 & 79.2 \\
$*$ & 59.4 & 1.8 & 33 & 95 & 70.1 \\
$\dagger$ & 54.0 & 1.8 & 30 & 86 & 58.9 \\
$\ddagger$ & 60.0 & 2.0 & 30 & 100 & 72 \\
\hline
\end{tabular}

Table 2

Schedules for advanced head and neck cancer. ${ }^{*}$ Accelerated schedule [52]; ${ }^{\dagger}$ Accelerated schedule [94]; ${ }^{\ddagger}$ Standard conventional schedule.

in mind is that acute responding tissues respond to radiotherapy by accelerated repopulation, which contributes to tissue sparing during fractionated radiotherapy. Thus, it is the late tissue response that is the dose limiting factor.

A clinical example which illustrates the BED concept is shown in Table 2 for advanced head and neck cancer. Three clinical accelerated fractionation schemes are outlined from O'Sullivan et al.[52] and Wratten et al. [94] as well as the standard treatment schedule. Within Table 2 we take $\alpha / \beta=20$ Gy for advanced head and neck cancer and $\alpha / \beta=3$ Gy for normal tissue.

Note, it is not feasible to compare Gy $y_{3}$ with Gy $y_{20}$ values, since the log cell kill obtained from Eq. 7 has been divided by $\alpha$. However, it is possible to compare toxicity to $\mathrm{Gy}_{3}$ values for normal tissue in different treatments and similarly evaluate the effectiveness of tumour cell kill in the different treatment strategies for $\mathrm{Gy}_{20}$ values. By comparing the overall dose of $60 \mathrm{~Gy}$ against the regimen for $54.9 \mathrm{~Gy}$, it can be seen that in the latter there is reduced toxicity to normal tissue.

One phase of the clinical trial by Wratten et al. had an overall dose of 54 Gy, but again comparing this against the other schedules in Table 2, shows that the impact of radiation treatment on the tumour is also greatly reduced. Accelerated radiotherapy for head and neck cancer has been assessed in randomized studies and it has been suggested that with this technique there is an increase in the severity of acute toxicity compared with that of conventional radiotherapy, (last row Table 2). In particular, O'Sullivan et al. noted that a schedule of total dose, $D=66$ Gy, 2 Gy per fraction for 33 fractions, was too severe for patients to tolerate and suggested to reduce the dose per fraction to $1.8 \mathrm{~Gy}$. More generally, it may be seen from the following example how knowledge of late-normal tissue and tumour $\alpha / \beta$ ratios is of major importance, in so far as the BED is a 
measure of how to design radiation treatment protocols, which might then lead to a better therapeutic ratio.

Example: Consider a treatment of head and neck cancer which delivers a total dose $D$, and let $d$ be the dose per fraction and $n=D / d$ the number of fractions. In the treatment schedule, a value of $\alpha / \beta=$ $3 G y$ is assumed for the healthy head and neck tissue and a value of $\alpha / \beta=20 G y$ is assumed for the head and neck tumour tissue and both tissues are exposed to the same overall dose $D$. In both cases, the biologically effective dose for the healthy tissue and tumour tissue are respectively

$$
\begin{aligned}
B E D_{3} & =D\left(1+\frac{d}{3}\right) \\
\text { and } B E D_{20} & =D\left(1+\frac{d}{20}\right)
\end{aligned}
$$

which are increasing functions of $d$. The smaller the dose per fraction, the better for the healthy head and neck tissue. An optimum treatment requires maximizing $B E D_{20}$ and minimizing $B E D_{3}$ and so it is necessary to consider $\max \left(B E D_{20}-B E D_{3}\right)$. That is, to examine the behaviour of

$$
B E D_{20}-B E D_{3}=D\left(\frac{d}{20}-\frac{d}{3}\right)=-\frac{17}{60} D d,
$$

which is decreasing in $d$. Thus, for smaller $d$ the difference between the healthy head and neck tissue and corresponding tumour tissue is increased which is the aim of a successful treatment protocol. The BED formula considered here do not take account of repopulation rates. This is considered in the next section.

\section{Repopulation and the LQ model}

In radiotherapy, treatment schedules are fractionated to allow the normal tissue to repair and recover from the irradiation. During these periods of recovery and resting, surviving clonogenic cells of the tumour also repair and repopulate. Saunders et al. reported that tumour cell repopulation occurring during a course of conventional radiotherapy may be the case of treatment failure [19]. Indeed, the nature of the re-growth of the particular tumour concerned is expected to influence the outcome of a specific treatment schedule $[32,66,29]$. Clinical radiation oncology treatment schedules also indicate how the effects of repopulation may be exploited to achieve improved tumour control [19,95,18,22,42,21,9].
Frequently, repopulation within the LQ model has been included in the very simple form based on the assumption of a time-dependent exponential term factored into the predicted clonogenic survival $[32,34,29]$. Such a model is in popular use and may be written in the form,

$\ln \sigma=-n\left(\alpha d+\beta d^{2}\right)-\lambda T$

where $T$ is the overall exposure time (i.e. the complete timescale of the treatment protocol) and $\lambda$ the exponential repopulation constant. An expression for $\lambda$ can be obtained by relating it to the clonogenic doubling time $T_{p}$. This allows Eq. 12 to be written as

$\ln \sigma=-n\left(\alpha d+\beta d^{2}\right)-\frac{T \ln 2}{T_{p}}$.

The model given by Eq. 13 was implemented by Wheldon et al. in 1977 to consider optimal uniform treatment schedules for cancer radiotherapy [32]. This was achieved by considering uniform treatment schedules and incorporation of radiation tolerance through the CRE (cumulative radiation effect) system. The CRE was developed by Kirk et al.[96] as a variation of the NSD (nominal standard dose) model. Equation 13 was also modified by Fowler to reflect the more realistic clinical setting in which there is a time delay, $T_{k}$, before repopulation is detectable [29]. As such Eq. 13 becomes

$\ln \sigma=-n\left(\alpha d+\beta d^{2}\right)-\frac{\left(T-T_{k}\right) \ln 2}{T_{p}}$.

It is typically assumed that repopulation starts at the onset time $T_{k}$ days and continues until the end of the radiotherapy schedule at $T$ days. Thus, the time available for cell repopulation is $T-T_{k}$ days. A constant doubling time of $T_{p}$ after $T_{k}$ days is assumed. Other similar repopulation models were considered in 1988 and 1989 by Wheldon and Amin[65] and Dale[67], and in 1995 Jones and Dale [12] studied the use of a time varying loss factor. This was represented by a mathematical function which declined exponentially either from the start of therapy or after some delay period.

These types of repopulation models, as given in Eq. 12-14, inherently assumes a constant tumour sensitivity and rate of growth of the tumour, i.e. exponential growth kinetics. However, it has been suggested by Ribba et al.[97] (and references therein) that cell cycle regulation and anti-growth signals such as hypoxia (Gray et al. [98]) can play an important role in the reduction in response to radiation. 


\begin{tabular}{|c|c|c|}
\hline \multicolumn{3}{|c|}{ Growth mechanism } \\
\hline$T_{p}$ Exponential & Logistic & Gompertz \\
\hline $301.059 \times 10^{-10}$ & $1.151 \times 10^{-10}$ & $3.517 \times 10^{-9}$ \\
\hline $607.129 \times 10^{-11}$ & $7.432 \times 10^{-11}$ & $4.688 \times 10^{-10}$ \\
\hline $906.248 \times 10^{-11}$ & $6.424 \times 10^{-11}$ & $2.263 \times 10^{-10}$ \\
\hline
\end{tabular}

That is, for those cells within the S-phase of the cell cycle, or given low levels of oxygenation, a higher level of radio-resistance occurs. During the course of treatment, re-distribution and re-oxygenation occurs which increases the net repopulation rate of the tumour $[99,100,39]$. Therefore the doubling time, $T_{p}$, is not constant, but dependent on the size of the tumour and it has been shown that larger tumours have longer volume doubling times than smaller ones $[101,102]$. One example of this may be found in some human lung cancers which have been shown by Steel to follow a Gompertzian pattern of growth [101,13]. Hence, the models presented so far may not be appropriate for all tumours.

In 1997 O'Donoghue considered a Gomp-ex model within an LQ formalism which assumed that a tumour follows a growth/re-growth curve which slows down as its size increases. Mathematically this model consisted of two equations, one which described the tumour to follow Gompertzian growth when the tumour was greater than a certain critical threshold size and the other that describes the tumour by an exponential equation when the tumour was less than the threshold size. O'Donoghue applied this to examine fractionated radiotherapy treatment [53]. Wheldon et al. have investigated the dose-response relationship for cancer incidence in a two stage radiation carcinogenesis model incorporating Gomp-ex cellular repopulation [54]. Lindsay et al. have applied the Gomp-ex model to study radiation carcinogenesis for risk of treatment-related second tumours following radiotherapy [55].

The authors of this article have also documented how the nature of repopulation can influence the outcome for a particular treatment schedule [40]. Table 3 illustrates our findings of the variation in outcome at the end of a treatment schedule resulting from the particular nature of the mechanism of repopulation. Indeed, the conclusions drawn were those that tumour following a repopulation mechanism of exponential or logistic growth resulted in similar outcomes, whilst those that followed a Gom- pertzian nature of repopulation resulted in a poorer prognosis for the patient. This was due to at least one order of magnitude more tumour cells surviving the treatment protocol which have then the potential to repopulate the tumour. Indeed, this effect is heightened by gaps in the treatment proto$\mathrm{col}$, whether these are planned or not. This leads to clinical implications depending on the different regrowth laws that may be acting during the course to radiation treatment and therefore should be considered during the clinical planning of radiation treatment of cancer.

Although repopulation is a significant factor to be considered within the LQ model, it still leaves redistribution and re-oxygenation to be dealt with. Brenner et al. considered this issue in 1995, and extended the LQ to that of the LQR model [36]. The LQR model includes the $4 \mathrm{R}$ 's of radiotherapy detailed by Withers[30], and deals with redistribution and reoxygenation through the concept of re-sensization, as detailed by Hlatky et al.[103]. The allowance of intra-tumour heterogeneity is essentially handled by considering a Gaussian distribution for $\alpha$ and $\beta$ and obtaining the mean SF. The LQR model is denoted by

$\ln \sigma=-\alpha d-\left(\beta-\frac{1}{2} \varsigma_{\alpha}^{2}\right) d^{2}$.

The form of the LQ model is preserved by the averaging and so the first term still denotes cell kill by one-track action, the second cell kill by two-track action (also incorporates repair), but now a term referring to cellular diversity is included, given by the dispersion about the mean radiosensitivity $\alpha$. Horas et al. have incorporated the LQR model into a 2compartment system for a tumour representing oxic and hypoxic zones [38]. These types of models are indeed the future direction of the LQ equation and its development, i.e. the inclusion of heterogeneity and diversity of the cellular structure of a tumour, as well as the nature of the type of repopulation.

\section{Tumour control probability models}

In this section we outline the development of more and more detailed models for the TCP. The Poissonian TCP model and the binomial TCP model are both based on the LQ model. In fact, any of the modifications that include repopulation and heterogeneity can also be used, e.g. Eq. 12-15. 
Let $n$ denote the number of tumor cells after treatment and $n_{0}$ the initial number of tumor cells. We assume that the cell number $n$ is a random variable with distribution $P(n)$. Then the TCP is the probability to have no tumor cells left, hence

$T C P=P(0)$.

We now assume that the surviving fraction $\sigma$ is a good estimator for $n / n_{0}$. If $n$ is Poisson distributed, then we get

$T C P=e^{-n_{0} \sigma}$

and if $n$ is binomial distributed we obtain

$T C P=(1-\sigma)^{n_{0}}$,

where $\sigma$ is given by one of Eq. $12-15$. Note that these TCP formulas coincide for large $n_{0}$ and small $\sigma$ (law of large numbers). Since these TCP formalisms are based on the LQ model, they show the same advantages and shortcomings. A strong advantage is its simplicity. The TCP and LQ models are based on the two parameters, $\alpha, \beta$, which are known for many tissues and cancer types. A disadvantage of these models is the fact that the time course of the treatment and the repopulation dynamics are not included, or are included artificially.

In a ground-breaking paper in 2000, Zaider and Minerbo developed a time dependent TCP model based on a stochastic birth-death process. In the end, the Zaider-Minerbo TCP formula (ZM) is based on the following differential equation model for the tumor cell number

$$
\begin{aligned}
\frac{d}{d t} N(t) & =(b-d-h(t)) N(t) \\
N(0) & =n_{0}
\end{aligned}
$$

where $b$ is the birth rate, $d$ the natural death rate and $h(t)$ the radiation induced death rate (hazard function). The treatment schedule is then explicitly included in the time dependence of $h(t)$. Based on Eq. 18 the TCP formula of ZM can be written as

$T C P(t)=\left(1-\frac{N(t)}{n_{0}+b n_{0} \int_{0}^{t} \frac{N(t)}{N(\tau)} d \tau}\right)^{n_{0}}$.

Note that in the case of no repopulation, $b=0$, we obtain the binomial TCP model from above Eq. 17.

The ZM-TCP formula explicitly uses exponential regrowth between treatments. This is, as shown by McAneney and O'Rourke [40] a good model for small tumor sizes. However, it would be interesting to study non-linear growth laws of the form

$\frac{d}{d t} N(t)=f(N)-d N-h(t) N$,

although it is very difficult to formulate and solve the corresponding nonlinear birth-death process. This might prevent the computation of an explicit TCP formula.

An extension of the ZM-model that includes cell cycle dynamics was developed by Dawson and Hillen [1]. It is known that quiescent cells (in the $G_{0}$-phase) are less radiosensitive than proliferating cells (in the $G_{1}, S, G_{2}, M$-phases). Dawson and Hillen split the tumor cell population into two compartments, active cells $A(t)$ and quiescent cells $Q(t)$ (if needed, more compartments could be considered). For the cell cycle dynamics we use a simple linear differential equation model that was proposed by Swierniak [104]. Combined with treatment we have

$$
\begin{aligned}
\frac{d}{d t} A(t) & =-b A-d A+\gamma Q-h_{a}(t) A \\
\frac{d}{d t} Q(t) & =2 b A-\gamma Q-d Q-h_{q}(t) Q \\
A(0) & =A_{0} \\
Q(0) & =Q_{0}
\end{aligned}
$$

where the new parameter $\gamma>0$ describes the transition from resting compartment into the cell cycle. Since quiescent cells are less radiosensitive, we assume $h_{a}(t)>h_{q}(t)$. Also for Eq. 20 the corresponding nonlinear birth-death process can be formulated and solved. This gives a quite complex TCP formula which we will not write down here, but we refer to Dawson and Hillen [1] for details.

In evaluating this new TCP formula we made the following observations:

- The DH-model should be used if a significant quiescent compartment is present. This is relevant for tumor spheroids with hypoxic interior.

- In general, the ZM-model overestimates the TCP, since it does not account for less radiosensitive cells.

- If the TCP models are used to compare different treatment schedules (as summarized in Table 1), then the ZM model and the DH model give very similar ranking. In general, a higher dose per fraction schedule seems to increase the TCP.

- A ranking based on the BED gives different ranking of schedules. As an example, the BED cannot distinguish between Schedule A: 2 Gy per day, 
5 days per week, 7 weeks, and Schedule B: 2 Gy twice a day, 5 days per week, 3.5 weeks. Whereas $\mathrm{ZM}$ and $\mathrm{DH}$ find that schedule $\mathrm{B}$ has a larger TCP which compares to the Schedule C: 4 Gy per day, 5 days per week, 3.5 weeks.

\section{Conclusions}

In this review we have examined the role of tumour repopulation kinetics in external beam radiotherapy within the LQ formalism and its influence on treatment outcome. An important feature of the tumour repopulation models considered here, are that they allow the effects of temporally nonuniform treatments to be described. Our work suggests that there may be untapped potential for the use of fractionation schemes that incorporate repopulation kinetics more closely aligned with the observed re-growth pattern of the particular tumour concerned. Our hope is that these types of models will provide a systematic method of refining existing approaches to improve the therapeutic index of cancer radiotherapy. Future work in this area will extend the repopulation models considered here to include the effects of re-oxygenation and re-distribution within the LQ formalism with a view to enhancing treatment outcome.

We have further considered the effects of the cell cycle within the context of the time-dependent TCP formalism of Zaider and Minerbo which incorporates a repopulation law based on a stochastic birth death process and shown that this can be used to analyze a variety of treatment plans of varying dose rates, number of fractions and overall treatment time. Inclusion of the cell-cycle is an important improvement on existing current TCP models in the literature and show it may be possible to manipulate the temporal structures of fractionation schedules to improve TCP outcome in cancer radiotherapy. Future work on the cell-cycle TCP model will include information about both hypoxia and re-oxygenation. In addition more complex patterns of repopulation will be taken into account. It is expected that inclusion of these extra factors will yield a more complete picture of tumour response to therapy.

\section{Acknowledgements}

Financial support is acknowledged by SFC O'Rourke and H McAneney from the Leverhulme Trust (Grant No. F/00 203/K).

\section{References}

[1] A. Dawson, T. Hillen, Derivation of the tumour control probability (tcp) from a cell cycle model, Comput. and Math. Meth. in Medicine 7 (2006) 121-142.

[2] J. F. Fowler, B. E. Stern, Dose-rate effects: some theoretical and practical considerations, Br. J. Radiol. 33 (1960) 389-395.

[3] H. R. Withers, H. D. Thames, L. J. Peters, A new isoeffect curve for change in dose per fraction, Radiother. Oncol. 1 (1983) 187-191.

[4] R. G. Dale, Dose-rate effects in targeted radiotherapy, Phys. Med. Biol. 41 (1996) 1871-1884.

[5] R. K. Sachs, D. J. Brenner, The mechanistic basis of the linear-quadratic model., Med. Phys. 25 (1998) $2071-2073$.

[6] T. E. Wheldon, C. Deehan, E. G. Wheldon, A. Barrett, The linear quadratic transformation of dose-volume histograms in fractionated radiotherapy, Radiother. Oncol. 46 (1998) 285-295.

[7] B. Jones, R. G. Dale, Mathematical models of tumour and normal tissue response, Acta Oncologica 38 (1999) 883-893.

[8] R. K. Sachs, L. R. Hlatky, P. Hahnfeldt, Simple ode models of tumour growth and anti-angiogenic or radiation treatment, Mathl. Comput. Modelling 33 (2001) 1297-1305.

[9] J. F. Fowler, Development of radiobiology for oncology - a personal view, Phys. Med. Biol. 51 (2006) R263R286.

[10] W. K. Sinclair, The shape of radiation survival curves of mammalain cells cultured in vitro., Biophysical Aspects of Radiation Quality, International Atomic Energy Agency, Technical Reports Series 58 (1966) 21-43.

[11] K. H. Chadwick, H. P. Leenhouts, A molecular theory of cell survival, Phys. Med. Biol. 18 (1973) 78-87.

[12] B. Jones, R. G. Dale, Cell loss factors and the linearquadratic model, Radiother. Oncol. 37 (1995) 136-139.

[13] G. G. Steel (Ed.), Basic Clinical Radiobiology, 3rd Edition, Arnold, 2002.

[14] E. J. Hall, A. J. Giaccia, Radiobiology for the Radiologist, sixth Edition, JB Lippincott Company, 2006.

[15] J. Horiot, R. L. Fur, T. N'Guyen, C. Chenal, S. Schraub, S. Alfonsi, G. Gardani, W. V. D. Bogaert, S. Danczak, M. Bolla, Hyperfractionation versus conventional fractionation in oropharyngeal carcinoma: Final analysis of a randomized trial of the eortc cooperative group of radiotherapy., Radiother. Oncol. 25 (1992) 231-241.

[16] C. C. Wang, Local control of oropharyngeal carcinoma after two accelerated hyperfractionated radiation therapy schemes, Int. J. Radiat. Oncol. Biol. Phys. 14 (1988) 1143-1146. 
[17] L. Peters, K. K. Ang, H. D. Thames, Accelerated fractionation in the radiation treatment of head and neck cancer: a critical comparison of different strategies, Acta Oncologica 27 (1988) 185-194.

[18] B. Maciejewski, K. Skladowskia, B. Pileckia, J. M. G. Taylor, R. H. Withersd, L. Miszczyka, A. Zajusza, R. Suwinskia, Randomized clinical trial on accelerated 7 days per week fractionation in radiotherapy for head and neck cancer. preliminary report on acute toxicity, Radiother. Oncol. 40 (1996) 137-145.

[19] M. I. Saunders, A. D. Barrett, M. K. Pamar, A. Harvey, A. Gibson, Randomized multicentre trials of chart $\mathrm{v}$ conventional radiotherapy in head and neck and nonsmall cell lung cancer, Br. J. Cancer 73 (1996) 14551462.

[20] S. M. Bentzen, M. I. Saunders, S. Dische, Repair halftimes estimated from observations of treatment-related morbidity after chart or conventional radiotherapy in head and neck cancer, Radiother. Oncol. 53 (1999) 219-226.

[21] D. D. Dionysiou, G. S. Stamatakos, Applying a $4 \mathrm{~d}$ multiscale in vivo tumor growth model to the exploration of radiotherapy scheduling: The effects of weekend treatment gaps and p53 gene status on the response of fast growing solid tumours, Cancer Informatics 2 (2006) 113-121.

[22] M. I. Saunders, A. D. Barrett, Continuous hyperfractionated accelerated radiotherapy (chart) versus conventional radiotherapy in non-small cell lung cancer: a randomized multicenter trial, Lancet 350 (1997) 161-165.

[23] E. B. Butler, B. S. Teh, W. H. Grant, B. M. Uhl, R. B. Kuppersmith, J. K. Chiu, D. T. Donovan, S. Y. Woo, Smart (simultaneous modulated accelerated radiation therapy) boost: a new accelerated fractionation schedule for the treatment of head and neck cancer with intensity modulated radiotherapy, Int. J. Radiat. Oncol. Biol. Phys. 45 (1999) 21-32.

[24] G. M. Duchesne, L. J. Peters, What is the $\alpha / \beta$ ratio for prostate cancer? rationale for hypofractionated highdose-rate brachytherapy, Int. J. Radiat. Oncol. Biol. Phys. 44 (1999) 747-748.

[25] D. J. Brenner, E. J. Hall, Fractionation and protraction for radiotherapy of prostate carcinoma, Int. J. Radiat. Oncol. Biol. Phys. 43 (1999) 1095-1101.

[26] J. F. Fowler, What hypofractionated protocols should be tested for prostate cancer?, Int. J. Radiat. Oncol. Biol. Phys. 56 (2003) 1093-1104.

[27] J. Overaaard, H. S. Hansen, W. Sapru, M. Overgaard, C. Grau, K. Jorgensen, L. Bastholt, O. Hansen, L. Specht, A. Berthelsen, M. Pedersen, Conventional radiotherapy as the primary treatment of squamous cell carcinoma of the head and neck. a randomized multicentre study of 5 versus 6 fractions per weekpreliminary report from the dahanca 6 and 7 trial, Radiother. Oncol. 40 (1996) S31.

[28] H. D. Thames, J. H. Hendry, Fractionation in Radiotherapy pp279, London: Taylor and Francis, 1987.

[29] J. F. Fowler, The linear-quadratic formula and progress in fractionated radiotherapy, Br. J. Radiol. 62 (1989) 679-694.
[30] H. R. Withers, The four r's of radiotherapy, Adv. Radiat. Biol. 5 (1975) 241-247.

[31] G. G. Steel, T. J. McMillan, J. H. Peacock, The 5rs of radiobiology, Int. J. Radiat. Biol. 56 (1989) 1045-1048.

[32] T. E. Wheldon, J. Kirk, J. S. Orr, Optimal radiotherapy of tumour cells following exponentialquadratic survival curves and exponential repopulation kinetics, Br. J. Radiol. 50 (1977) 681-682.

[33] J. R. Usher, Mathematical derivation of optimal uniform treatment schedule for the fractionated irradiation of human tumours, Math. Biosc. 49 (1980) 157-184.

[34] E. L. Travis, S. L. Tucker, Isoeffect models and fractionated radiation therapy, Int. J. Radiat. Biol. 13 (1987) 283-287.

[35] B. G. Woulters, J. M. Brown, Cells at intermediate oxygen levels can be more important than the hypoxic fraction in determining tumour response to fractionated radiotherapy., Radiat. Res. 147 (1997) $541-550$.

[36] D. J. Brenner, L. R. Hlatky, P. J. Hahnfeldt, E. J. Hall, R. K. Sachs, A convenient extension of the linear-quadratic model to include redistribution and reoxygenation, Int. J. Radiat. Oncol. Biol. Phys. 32 (1995) 379-390.

[37] F. M. Buffa, C. West, K. Byrne, J. V. Moore, A. E. Nahum, Radiation response and cure rate of human colon adenocarcinoma spheroids of different size: the significance of hypoxia on tumour control modelling, Int. J. Radiat. Oncol. Biol. Phys. 49 (2001) 1109-1118.

[38] J. A. Horas, O. R. Olguin, M. G. Rizzotto, On the surviving fraction in irradiated multicellular tumour spheroids: calculation of overall radiosensitivity parameters, influence of hypoxia and volume effects, Phys. Med. Biol. 50 (2005) 1689-1701.

[39] L. M. Wein, J. E. Cohen, J. T. Wu, Dynamic optimization of a linear-quadratic model with incomplete repair and volume-dependent sensitivity and repopulation, Int. J. Radiat. Biol. Phys. 47 (2000) 1073-1083.

[40] H. McAneney, S. F. C. O'Rourke, Investigation of various growth mechanisms of solid tumour growth within the linear quadratic model for radiotherapy, Phys. Med. Biol. 52 (2007) 1039-1054.

[41] V. P. Antipas, G. S. Stamatakos, N. K. Uzunoglu, D. D. Dionysiou, R. G. Dale, A spatio-temporal simulation model of the response of solid tumours to radiotherapy in vivo: parametric validation concerning oxygen enhancement ratio and cell cycle, Phys. Med. Biol. 49 (2004) 1485-1504.

[42] D. D. Dionysiou, G. S. Stamatakos, N. K. Uzunoglu, K. S. Nikita, A. Marioli, A four-dimensional simulation model of tumour response to radiotherapy in vivo: parametric validation considering radiosensitivity, genetic profile and fractionation, J. Theor. Biol. 230 (2004) $1-20$.

[43] P. L. Chen, D. J. Brenner, R. K. Sachs, Ionizing radiation damage to cells: Effects of cell cycle resdistribution, Math. Biosci. 126 (1995) 147-170.

[44] P. Hahnfeldt, L. Hlatky, Resensitization due to redistribution of cells in the phases of the cell cycle 
during arbitrary radiation protocols., Radiat. Res. 145 (1996) 134-143.

[45] L. E. Dillehay, A model of cell killing by low-dose radiation including repair of sub-lethal damage, $g_{2}$ block, and cell division., Radiat. Res. 124 (1990) 201207.

[46] M. Zaider, C. S. Wuu, G. N. Minerbo, The combined effects of sublethal damage repair, cellular repopulation and redistribution in the mitotic cycle. i survival probabilities after exposure to radiation., Radiat. Res. 145 (1996) 457-466.

[47] C. I. Armpilia, R. G. Dale, B. Jones, Determination of the optimum dose per fraction in fractionated radiotherapy when there is delayed onset of tumour repopulation during treatment, Br. J. Radio. 77 (2004) 765-767.

[48] J. P. Kirkpatrick, L. B. Marks, Modelling killing and repopulation kinetics of subclinical cancer: direct calculations from clinical data, Int. J. Radiat. Oncol. Biol. Phys. 58 (2004) 641-654.

[49] J. J. Kim, I. F. Tannock, Repopulation of cancer cells during therapy: an important cause of treatment failure, Nature Reviews: Cancer 5 (2005) 516-525.

[50] J. F. Fowler, Biological factors influencing optimum fractionation in radiotherapy, Acta Oncologica 40 (2001) $712-717$.

[51] J. F. Fowler, R. Chappell, Non-small cell lung tumours repopulate rapidly during radiation therapy., Int. J. Radiat. Oncol. Biol. Phys. 46 (2000) 516-517.

[52] J. M. O'Sullivan, D. P. Hollywood, N. Cody, J. Dillon, S. Buckney, M. J. Moriarty, J. G. Armstrong, Accelerated radiation therapy, seven fractions per week, for advanced head and neck cancer-a feasibility study., Clin. Oncol. (R Coll Radiol) 14 (2002) 236-240.

[53] J. A. O'Donoghue, The response of tumours with gompertzian growth characteristics to fractionated radiotherapy, Int. J. Radiat. Biol. 72 (1997) 325-339.

[54] E. G. Wheldon, K. A. Lindsay, T. E. Wheldon, The dose response relationship for cancer incidence in a two-stage radiation carcinogenesis model incorporating cellular repopulation., Int. J. Radiat. Biol. 76 (2000) 699-710.

[55] K. A. Lindsay, E. G. Wheldon, C. Deehan, T. E. Wheldon, Radiation carcinogenesis modelling for risk of treatment-related second tumours following radiotherapy, Br. J. Radiol. 74 (2001) 529-536.

[56] J. H. Mao, K. A. Lindsay, R. J. Mairs, T. E. Wheldon, The effect of tissue-specific growth patterns of target stem cells on the spectrum of tumours resulting from multistage tumorigenesis, J. Theor. Biol. 210 (2001) 93-100.

[57] T. R. Munro, C. W. Gilbert, The relation between tumor lethal doses and the radiosensitivity of tumor cells, Br. J. Radiol. 34 (1961) 246-251.

[58] E. H. Porter, The statistics of dose-cure relationships for irradiated tumors. part i and ii, Br. J. Radiol. 53 (1980) 210-227 and 336-345.

[59] A. Brahme, Dosimetric precision requirements in radiation therapy, Acta Radiol. Oncol. 23 (1984) 379391.

[60] J. D. Brenner, Dose, volume and tumour control predictions in radiotherapy, Int. J. Radiat. Oncol. Biol. Phys. 26 (1993) 171-179.
[61] S. L. Tucker, H. D. Thames, J. M. G. Taylor, How well is the probability of tumor cure after fractionated irradiation described by poisson statistics?, Radiat. Res. 124 (1990) 273-282.

[62] A. Y. Yakovlev, Comments on the distribution of clonogens in irradiated tumors, Radiat. Res. 134 (1993) $117-120$.

[63] W. S. Kendal, A closed form description of tumour control with fractionated radiotherapy and repopulation., Radiat. Biol. 73 (1998) 207-210.

[64] S. L. Tucker, J. M. G. Taylor, Improved models of tumor cure, Int. J. Radiol. Biol. 70 (1996) 539-553.

[65] T. E. Wheldon, A. E. Amin, The linear-quadratic model, Br. J. Radiol. 61 (1988) 700-702.

[66] H. R. Withers, Some changes in concepts of dose fractionation over 20 years., in: J. M. Vaeth, J. Meyer (Eds.), Time, dose and Fractionation in the Radiation Therapy of Cancer Frontiers of Radiation Therapy and Oncology, Vol. 22, karger, Basel, 1988, pp. 1-13.

[67] R. G. Dale, Time-dependent tumour repopulation factors in linear quadratic equations - implications for treatment strategies., Radiother. Oncol. 15 (1989) 371382.

[68] W. A. Tomé, J. Fowler, On the inclusion of proliferation in tumour control probability calculations for inhomogeneously irradiated tumours, Phys. Med. Biol. 48 (2003) N261-N268.

[69] A. Niemierko, M. Goitein, Implementation of a model for estimating tumour control probability for an inhomogeneously irradiated tumor, Radiother. Oncol. 29 (1993) 140-147.

[70] J. D. Fenwick, Predicting the radiation control probability of heterogeneous tumour ensembles: data analysis and parameter estimation using a closed form expression, Phys. Med. Biol. 43 (1998) 2159-2178.

[71] A. E. Nahum, D. M. Tait, Maximising control by customized dose prescription for pelvic tumours, in: A. Breit (Ed.), Advanced Radiation Therapy: Tumour Response Monitoring and Treatment Planning, Heidelberg: Springer, 1992, pp. 425-431.

[72] S. Webb, A. E. Nahum, A model for calculating tumour control probability in radiotherapy including the effects of inhomogeneous distributions of dose and clonogenic cell density, Phys. Med. Biol. 38 (1993) 653-666.

[73] S. Webb, Optimum parameters in a model for tumour control probability including interpatient heterogeneity, Phys. Med. Biol. 39 (1994) 1895-1914.

[74] R. Mohan, G. S. Mageras, B. Baldwin, L. J. Brewster, G. J. Kutcher, Clinically relevant optimisation of 3d conformal treatments, Med. Phys. 19 (1992) 933-944.

[75] M. Zaider, G. N. Minerbo, Tumour control probability: a formulation applicable to any temporal protocol of dose delivery, Phys. Med. Biol. 45 (2000) 279-293.

[76] P. Stavrev, M. W. B. Warkentin, N. Stavreva, B. G. Fallone, Radiation damage, repopulation and cell recovery analysis of in vitro tumour cell megacolony culture data using a non-poissonian cell repopulation tcp model, Phys. Med. Biol. 50 (2003) 3053-3061.

[77] G. J. Kutcher, C. Burman, L. Brewster, M. Goitein, R. Mohan, Histogram reduction method for calculating complication probabilities for three dimensional treatment planning evaluations, Int. J. Radiat. Oncol. Biol. Phys. 21 (1991) 137-146. 
[78] A. Niemierko, M. Goitein, Calculation of normal tissue complication probability and dose-volume histogram reduction schemes for tissues with a critical element architecture, Radiother. Oncol. 20 (1991) 166-176.

[79] R. $\mathrm{K}$. Sachs, P. Hahnfeld, D. J. Brenner, The link between low-let dose-response relations and the underlying kinetics of damage production/repair/misrepair, Int. J. Radiat. Biol. 72 (1997) 351-374.

[80] D. Brenner, L. R. Hltaky, P. J. Hahnfeldt, Y. Huang, R. K. Sachs, The linear quadratic and most other common radiobiogical models, Radiat Res 150 (1998) $83-88$.

[81] M. Guerrero, R. D. Stewart, J. Wang, X. A. Li, Equivalence of the linear-quadratic and two-lesion kinetic models, Phys. Med. Biol. 47 (2002) 3197-3209.

[82] D. E. Lea, Actions of Radiations on Living Cells, London: Cambridge University Press, 1946.

[83] A. M. Kellerer, H. H. Rossi, The theory of dual radiation action, Current Topics in Radiation Research Quarterly 8 (1972) 85-158.

[84] K. H. Chadwick, H. P. Leenhouts, The molecualr theory of radiation biology, Springer, Berlin, 1981.

[85] C. A. Tobias, The repair-misrepair model in radiobiology, comparison to other models., Radiat. Research 8 (1985) S77-S95.

[86] R. G. Dale, The application of the linear-quadratic dose-effect equation to fractionated and protracted radiotherapy, Br. J. Rad. 58 (1985) 515-528.

[87] S. B. Curtis, Lethal and potentially lethal lesions induced by radiation - a unified repair model, Radiat. Res. 106 (1986) 252-270.

[88] J. S. Bedford, M. N.Cornforth, Relationship between recovery from sublethal x-ray damage and the rejoining of chromosome breaks in normal human fibroblasts, Radiat. Research 111 (1987) 406-423.

[89] D. Harder, The pairwise lesion interaction model, in: J. Kiefer (Ed.), Quantitative Mathematical Models in Radiative Biology, Berlin and New York: SpringerVerlag, 1988, pp. 159-170.

[90] H. D. Thames, S. M. Bentzen, I. Turesson, M. Overgaard, W. V. den Bogaert, Time-dose factors in radiotherapy: a review of the human data, Radiother. Oncol. 19 (1990) 219-235.

[91] D. J. Carlson, R. D. Stewart, X. A. Li, K. Jennings, J. Z. Wang, M. Guerro, Comparison of in vitro and in vivo $\alpha / \beta$ ratios for prostate cancer, Phys. Med. Biol. 49 (2004) 4477-4491.

[92] C. R. King, T. A. DiPetrillo, D. E. Wazer, Optimal radiotherapy for prostate cancer: predictions for conventional external beam, imrt, and brachytherapy from radiobiologic models, Int. J. Radiat. Oncol. Biol. Phys. 46 (2000) 165-172.

[93] G. W. Barendsen, Dose fractionation, dose rate and iso-effect relationships for normal tissue responses., Int. J. Radiat. Oncol. Bio. Phys. 8 (1982) 1981-1997.

[94] C. R. Wratten, M. G. Poulsen, S. Williamson, Tripcony, J. Keller, G. Dickie, Effect of surgery on normal tissue toxicity in patients treated with accelerated radiotherapy, Acta Oncologica 41 (2002) 56-62.

[95] B. Maciejewski, S. Majewski, Dose fractionation and tumour repopulation in radiotherapy for bladder cancer, Radiother. Oncol. 21 (1991) 163-170.
[96] J. Kirk, W. M. Gray, E. R. Watson, Cumulative radiation effect. part 1: Fractionated treatment regimes, Clin. Radiol. 22 (1971) 145-155.

[97] B. Ribba, T. Cloin, S. Schnell, A multiscale model of cancer, and its use in analyzing irradiation therapies, Theor. Biol. Med. Mod. 3 (2006) 1-19.

[98] L. H. Gray, A. D. Conger, M. Ebert, S. Hornsey, O. C. Scott, The concentration of oxygen dissolved in tissues at the time of irradiation as a factor in radiotherapy, Br. J. Radiol. 26 (1953) 638-648.

[99] J. F. Fowler, The phantom of tumor treatment continually rapid proliferation unmasked, Radiother. Oncol. 22 (1991) 156-158.

[100] E. Sham, R. E. Durand, Cell kinetics and repopulation during multifraction irradiation of spheriods, Radiother. Oncol. 46 (1998) 201-207.

[101] G. G. Steel, Growth Kinetics of Tumours, Oxford: Clarendon Press, 1977.

[102] J. A. Spratt, D. von Fournier, J. S. Spratt, E. E. Weber, Decelerating growth and human breast cancer., Cancer 71 (1993) 2013-2019.

[103] L. R. Hlatky, P. Hahnfeldt, R. K. Sachs, Influence of time dependent stochastic heterogeneity on the radiation response of a cell population, Math. Biosci. 122 (1994) 201-220.

[104] A. Swierniak, A. Polanski, M. Kimmel, Optimal control problems arising in cell-cycle-specific cancer chemotherapy., Cell Prolif. 29 (1996) 117-139. 